\title{
Increasing Incidence of Colorectal Cancer, Starting at a Younger Age for Rectal Compared to Colon Cancer in Brunei Darussalam
}

\author{
Vui Heng Chong ${ }^{1 *}$, Pemasari Upali Telisinghe ${ }^{2}$, Ian Bickle ${ }^{3}$, Muhamad Syafiq \\ Abdullah ${ }^{1}$, Ediwn Lim ${ }^{2}$, Chee Fui Chong ${ }^{3}$
}

\begin{abstract}
Background: Colorectal cancer (CRC) is the most common gastrointestinal malignancy and is a significant cause of mortality. Its incidence is generally increasing in Asia. Reports from the West have indicated that the incidence of rectal cancer is increasing in the younger population. This study assessed the time trend of CRC in Brunei Darussalam specifically assessing the different age groups at which the incidences start to increase. Materials and Methods: The National Cancer registry was reviewed (1991 to 2014). The age standardized rate (ASR) and the age specific incidence rates (ASIRs) for three time periods (1991-1998), (1999-2006) and (2007-2014) were calculated. Results: The mean age of diagnosis was $59.3 \pm 14.6$ years old, incidences being slightly higher amongst men $(57.6 \%)$ and Malays $(67.1 \%)$. The most common tumor type was adenocarcinoma $(\mathbf{9 6 . 4 \%})$. Rectal cancers accounted for $35.2 \%(n=372 / 1,056)$ of all cancers of the large bowel; more men were affected than women. The proportion of rectal cancer was also high among the indigenous group. In the three time periods, the ASR for CRC increased from 16 per 100,000 (1991-1998) to 19.6 per $100,000(1999-2006)$ and 24.3 per 100,000 (2007-2014). The ASIRs for CRC increased markedly between the time periods 1998-2006 and 2007-2014, beginning in the 40-44 years age group. For rectal cancers, the ASIRs started to increase in the 25-29 age group onward whereas for colon cancers, the increase was observed at a later age, starting from the 45-49 age group. Conclusions: Our study showed an increase in the incidence of CRC including in the younger age groups. The increase was seen earlier in rectal cancer compared to colon cancer. These data mirror the trends reported from the West.
\end{abstract}

Keywords: Rectal cancer - colorectal neoplasms - time trend - incidence - Brunei Darussalam

Asian Pac J Cancer Prev, 16 (12), 5063-5067

\section{Introduction}

Colorectal cancer (CRC) is the most common cancer of the gastrointestinal tract and is the third most common cancer in men $(746,000$ cases, $10.0 \%$ of the total cancers $)$ and the second in women $(614,000,9.2 \%$ of the total cancers) worldwide (IARC 2012). It remains an important cause of morbidity and mortality worldwide and the incidence continue to increase (IARC, 2012; Zheng et al., 2014). In countries where there are CRC screening programs, the actual incidence has been reported to have stabilized and even declined as a result of early detection and prevention of progression to $\mathrm{CRC}$ through removal of premalignant lesions (IARC, 2012; Austin et al., 2014).

Earlier data from the United States based on the SEER (1973-1999) data have reported the incidence of colon cancer to have already stabilized, and declining by $11 \%$ to $72.1 / 100,000$ persons. However, the incidence of young colon and rectal cancers increased significantly by $17 \%$ (ASR 2.1/100,000) and 75\% $(1.4 / 100,000)$ respectively.
A recent study utilizing the same database (1975-2010) demonstrated that this trend continued, confirming that the trend is real (Bailey et al., 2014). The latter study reported that the incidence rates of localized, regional and distant colon and rectal cancers had increased for those aged between 20 and 34 years old. Specifically for rectal cancer, this was observed in the slightly older aged group, 35 to 49 years (Bailey et al., 2014). Such trends are of concerns considering that people in this age range are in the prime of their life and economically most active, contributing to the economy. However, not every developed nation is reporting such a trend. In Japan, although there have been an increase in the incidence of colon and rectal cancers, the increase is attributed to a cohort effect (Minami et al., 2006), rather than an actual increase in young colon and rectal cancers.

We previously reported that the incidence of CRC has been increasing in Brunei Darussalam (Chong et al., 2009). However, this study did not assess in detail the age groups when the incidence starts to increase. It is 


\section{Vui Heng Chong et al}

uncertain whether the trends reported from the west will also be observed in the east. Given the differences in the population demographic, especially in the genetic makeup, race/ethnicities along with the differences in social, culture, diet and level of development of a country, it is important to know if there are any differences in the trends of CRC for healthcare planning. Therefore, this study was done to assess the trends among the different age groups for both colon and rectal cancer in Brunei Darussalam, a developing South-East Asian nation.

\section{Materials and Methods}

\section{Setting}

Brunei Darussalam is a developing nation located on the northeast corner of the Borneo Island in South-East Asia. It has an estimated population of 420,000 (2012 Census) with Malays being the predominant group (68\%), followed by the Expatriates $(\sim 20 \%)$, Chinese $(11 \%)$ and the Indigenous group $(<5 \%)$. The health coverage is provided free to the Citizens and permanent residents (Locals) and payment is required for the Expatriate groups. Each district has a government hospital served by multiple strategically located health centers or clinics. The only tertiary referral center (RIPAS Hospital), a 550 bedded hospital covering all subspecialties is located in the Capital (Bandar Seri Begawan) and houses the State Pathology Laboratory (Department of Pathology), which receives and processes all tissue specimens from all the health institutions and centers within the country. A cancer registry which captures all tissue proven malignancies is maintained by the Department of Pathology, RIPAS Hospital from 1984 to the present day.

\section{Patients}

Patients with histology proven rectal carcinoma diagnosed between 1991 and 2014 were identified from the Department of Pathology cancers registry (RIPAS Hospital). This cancer registry was started in 1984, however data on age was not available for 1984 and 1985, and data on the location of large bowel tumors was incomplete in the years before 1991, therefore, these were excluded from analyses.

\section{Data}

Data on patient demographic (age, gender and race) and tumor (type of tumor) data was extracted.

\section{Statistical analyses}

The age standardized rate (ASR) and Age Specific Incidence Rates (ASIRs) for three time periods (19911998, 1999-2006 and 2007-2014 were calculated based on the projected population for Brunei (Population census department, Ministry of Finance) and the world population (WHO data). The population breakdowns of 1994, 2003 and 2010 were used to calculate the ASR and ASIRs. The ASIRs for CRC, rectal cancer and colon cancer were also calculated. The data were coded and entered into the Word Excel program and later entered into the SPSS (version 16.0, Chicago, IL, USA) for analysis.

\section{Results}

Over the study period (1991-2014), there were 1,057 cases of CRCs histologically diagnosed. Table 1 shows the demographic of the overall colorectal, rectal and colon cancer cases.

The mean age of diagnosis was $59.3 \pm 14.6$ years old; for rectum $59.3 \pm 14.4$ years old and colon $59.3 \pm 14.7$ years old. For colon cancer, there was no difference in the mean age of diagnosis between the genders, but older among the indigenous and the Chinese.

Over the three time period, the overall ASR increased from 16 per 100,000 (1991-1998) to 19.6 per 100,000 in 1999-2006 and 24.3 per 100,000 in 2007-2014. The ASR for colorectal cancer over the three time periods is shown in Figure 1.

Between the three time periods, apart from minor fluctuation in the 40-44 age groups, the ASIRs increased with each time period. The increase between the time period 2007-2014 and 1999-2006 was marked with the exception of the older age groups ( $>70$ years old) where the ASIR in 1999-2006 was slightly higher than 20072014 (Figure 2).

\section{Rectal cancers}

For rectal cancer, the mean age of diagnosis was younger among females $(57.8 \pm 14.4)$ compared to males $(60.3 \pm 14.1, p=0.107)$ and older among the Chinese $(60.4 \pm 12.7)$ compared to the Malays $(59.9 \pm 15.2)$,

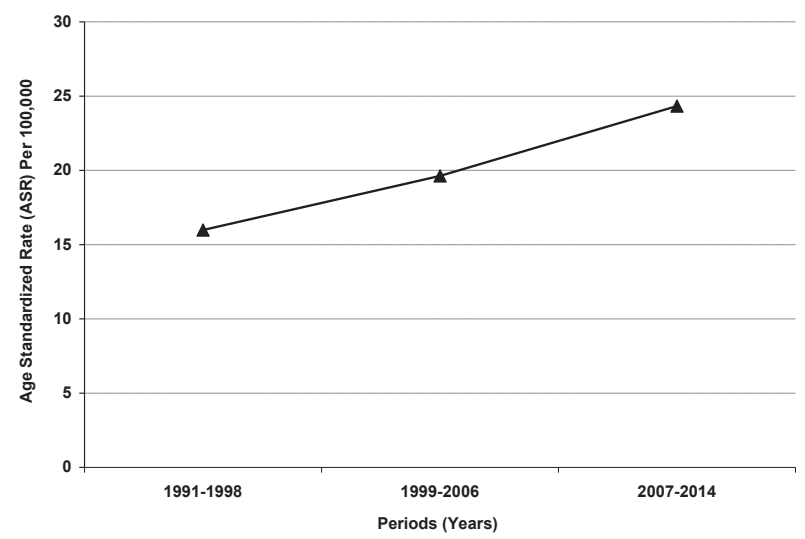

Figure 1. The ASR for Colorectal Cancer Over the Three Time Period

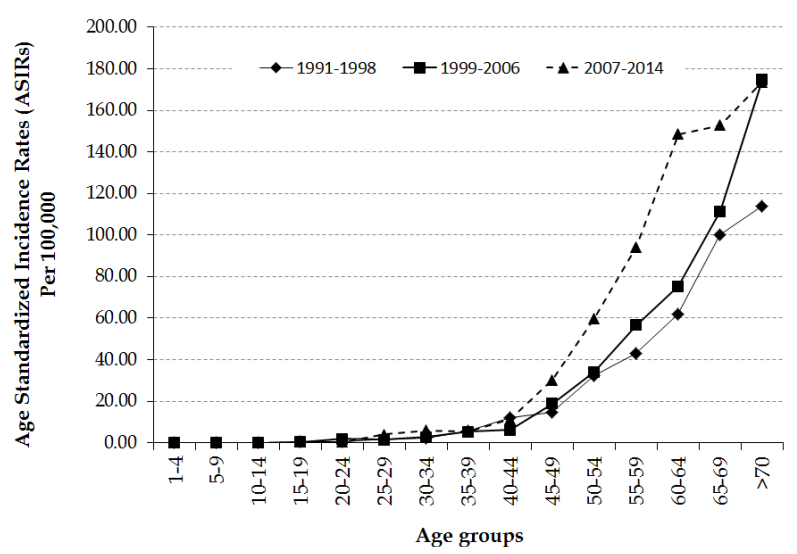

Figure 2. The ASIRs for Colorectal Cancer for the Three Time Periods 
Increasing Incidence of Colorectal Cancer, Starting at a Younger Age for Rectal Cancer in Brunei Darussalam

Table 1. Demographic of Colorectal Cancers; Overall, Rectal and Colon Cancers

\begin{tabular}{|c|c|c|c|}
\hline Variables & $\begin{array}{l}\text { Rectal cancer } \\
\qquad \begin{array}{c}\mathrm{N}=372) \\
\mathrm{n}(\%)\end{array}\end{array}$ & $\begin{array}{l}\text { Colon cancer } \\
\begin{array}{c}(\mathrm{N}=685) \\
\mathrm{n}(\%)\end{array}\end{array}$ & $\begin{array}{c}\text { Overall } \\
\mathrm{N}=1057 \\
\mathrm{n}(\%)\end{array}$ \\
\hline \multicolumn{4}{|l|}{ Gender } \\
\hline Male & $228(61.3)$ & $381(55.6)$ & 609 (57.6) \\
\hline Female & 144 (38.7) & 304 (44.4) & $448(42.4)$ \\
\hline \multicolumn{4}{|l|}{ Race } \\
\hline Malays & $256(68.8)$ & $453(66.2)$ & $709(67.1)$ \\
\hline Chinese & $82(22.0)$ & $162(23.7)$ & $244(23.1)$ \\
\hline Indigenous & $12(3.2)$ & $12(1.8)$ & $24 \quad(2.3)$ \\
\hline Others & $22 \quad(5.9)$ & $57 \quad(8.3)$ & $79 \quad(7.5)$ \\
\hline \multicolumn{4}{|l|}{ Tumor type } \\
\hline Adenocarcinoma & $357(96.0)$ & $662(96.6)$ & 1019 (96.4) \\
\hline Neuroendocrine & $9(2.4)$ & $7(1.0)$ & $16(1.5)$ \\
\hline Anaplastic & $2(0.5)$ & $0 \quad(0)$ & $2(0.2)$ \\
\hline Lymphoma & $1 \quad(0.3)$ & $9 \quad(1.3)$ & $10(0.9)$ \\
\hline GIST & $1 \quad(0.3)$ & $2(0.3)$ & $3(0.3)$ \\
\hline Signet cell & $1 \quad(0.3)$ & $1 \quad(0.1)$ & $2(0.2)$ \\
\hline Squamous cell & $1 \quad(0.3)$ & $0 \quad(0)$ & $1(0.1)$ \\
\hline Liposarcoma & $0 \quad(0)$ & $2(0.3)$ & $2(0.2)$ \\
\hline
\end{tabular}

Table 2. The Breakdown of the Age Specific Incidence Rates (ASIRs) for Rectal Cancer for the Different Age Groups Over the Three Period Blocks

\begin{tabular}{llcl}
\hline Time periods & $1991-1998$ & $1999-2006$ & $2007-2014$ \\
\hline Age groups & 0 & 0 & 0 \\
$1-4$ & 0 & 0 & 0 \\
$5-9$ & 0 & 0 & 0 \\
$10-14$ & 0.51 & 0 & 0.37 \\
$15-19$ & 0 & 0 & 0.36 \\
$20-24$ & 0.87 & 0.38 & 2 \\
$25-29$ & 0 & 1.19 & 1.75 \\
$30-34$ & 1.02 & 0.44 & 3.17 \\
$35-39$ & 3.54 & 1.45 & 4.7 \\
$40-44$ & 5.71 & 3.67 & 12.56 \\
$45-49$ & 7.66 & 11.39 & 19.3 \\
$50-54$ & 19.45 & 21.65 & 38.11 \\
$55-59$ & 20.66 & 32.98 & 57.39 \\
$60-64$ & 25.04 & 40.2 & 48.28 \\
$65-69$ & 41.14 & 49.59 & 66.6 \\
$>70$ & & & \\
\hline
\end{tabular}

Table 3. The Breakdown of the Age Specific Incidence Rates (ASIRs) for Colon Cancer in the Different Age Groups Over the Three Period Blocks

\begin{tabular}{lccc}
\hline Time periods & $1991-1998$ & $1999-2006$ & $2007-2014$ \\
\hline Age groups & & & \\
$1-4$ & 0 & 0 & 0 \\
$5-9$ & 0 & 0 & 0 \\
$10-14$ & 0 & 0.41 & 0 \\
$15-19$ & 0 & 1.95 & 0 \\
$20-24$ & 0.47 & 1.14 & 0 \\
$25-29$ & 0.87 & 1.58 & 2 \\
$30-34$ & 2.17 & 4.86 & 4.2 \\
$35-39$ & 4.58 & 4.84 & 2.38 \\
$40-44$ & 8.49 & 15.28 & 6.58 \\
$45-49$ & 9.13 & 22.79 & 17.79 \\
$50-54$ & 24.51 & 34.97 & 40.66 \\
$55-59$ & 23.77 & 42.4 & 56.05 \\
$60-64$ & 41.32 & 71.13 & 91.04 \\
$65-69$ & 75.11 & 125.06 & 104.61 \\
$>70$ & 72.6 & & 107.03 \\
\hline
\end{tabular}

Indigenous 58.2 \pm 13.4$)$ and the Others $(52.4 \pm 10.6)$ (ANOVA, $\mathrm{p}=0.126$ for trend). There were more males affected $(61.3 \% ; 38.7 \%)$ and the predominant tumor type was adenocarcinoma.

The Age Specific Incidence rates (ASIRs) for rectal cancer is shown Table 2 . The incidence started to increase after the 20-24 years age group, becoming more obvious in the 25-29 age groups onwards.

\section{Colon cancers}

The summary of the colon cancer is shown in Table 1. The ASIRs for colon cancer also showed an increase in the incidence, but this is only seen beginning from the 45-49 groups onward up until the 65-69 age groups. Generally, the increase in the ASIRs for colon cancer was not consistent with minor fluctuations between the time periods (Table 3 ).

\section{Discussion}

The characteristic of our patients with CRC, male predominant, tumor type (adenocarcinoma) and locations (rectum 35.2\% and colon 64.8\%) are similar to what have been reported regionally and globally (IARC, 2012; Austin et al., 2014; Bailey et al., 2014; Cai et al., 2014, Elsamany et al., 2914, Lim, 2014, Magaji et al., 2014). However, the mean age of diagnosis in our setting is much younger than those reported in the West. Our mean age of diagnosis is generally comparable to what have been reported in the region (Lim, 2014). We previously reported that young CRC is common in our setting (Koh et al., 2015) and this is reflected in our finding of a younger mean age of diagnosis. For rectal cancer, the mean age of diagnosis was younger among females compared to males, but not significantly different. Among the racial groups, the Others group was the youngest, but again, this was not significant. For rectal cancer, men were more affected compared to colon cancer. Among the various racial groups, the indigenous group had the highest proportion of rectal cancer. For colon cancer, apart from the gender differences and just slightly more men affected, the demographic are similar to rectal cancers.

Our study showed that the incidence (ASR) of CRC increased between the three time periods $0.45 \%$ per year in the first time period (1991-1998) to $0.59 \%$ per year in the second time period (2007-2014). The ASIRs increased consistently between the three time periods apart from minor fluctuation, whereas the rate in the 40-44 age groups was higher in the 1991-1998 period compared to the 1999-2006 period. The ASIRs for 20072014 were consistently higher than the two time periods beginning in the $40-44$ year age group. These increase are not unexpected considering that the population has been steadily increasing and growing older. Our findings are similar to that been reported in countries where the incidences of CRC are still increasing (IARC 2012). However, the increase are variable depending on the age groups, bigger and more obvious in the older age groups. What is of concern is the rate of increase between the different time periods. The increase was more marked between 1999-2006 and 2007-2014, compared to 1991- 
1998 and 1999-2006, with the exception of the older age group. If the pace of increase continues, the number of CRC cases may suddenly overwhelm the healthcare system. Therefore healthcare planning needs to take in account this possibility.

The increase in the ASR and ASIRs varies between countries, and generally correlate with the level of development of the country and this is also reflected in the increasing life expectancy of the particular population. Generally, the more developed a country, the higher the incidence of CRC (IARC, 2012). In the more developed nations of the West, the rates have actually been decreasing. Increasing trends which are now being seen in the developing nations have already peaked several decades back in the developed nations. Therefore, there trends in the developing nations are lagging behind the trends reported in the West by several decades (IARC, 2014).

Based on the data from the International Agency for Research on Cancer (IARC 2012), the ASR of CRC reported for men are as follows; Japan (just above $20 / 100,000$ in the late 1970 s, peaked and doubled in the early 1990s), Singapore ( 25/100,000 in the 1970s to just above $40 / 100,000$ in 2003 with the rate leveling off), China (increasing just above 26/100,000 in 1990 to $35 / 100,000$ in 2006$)$ and Thailand $(\sim 7.0 / 100,000$ in 1985 to $\sim 14.0 / 100,000$ in 2006). In Australia and New Zealand, the rates of CRC for men peaked at almost $50 / 100,000$ and $\sim 53 / 100,000$ respectively, and are now declining, following the trends seen in the developed nations of the West. For women, the trends are similar with the exceptions of the overall rates being $20 \%$ to $30 \%$ lower than their male counterparts. Similarly for women, the rates have stabilized and are actually declining in the developed nations and increasing in the developing nations (IARC, 2012). These variable rates increase in the incidence between countries in part depends on the baseline rates which is influenced by the genetic and racial make-up of the population, dietary habit and also environmental factors.

Among rectal cancers in our population, the ASIRs increased between the three time periods and the trend started at a younger age group compared to colon cancer. The definite increase and divergence (ASIRs for rectal cancer) started at the 25-29 age groups onward, whereas for colon cancer this was only seen from the 45-49 groups onward. There were some fluctuations in the rates, being slightly higher in the 20-24 years age group for rectal cancer and 30-34 year age group for colon cancer. These perhaps reflected the background fluctuations. Therefore, our findings are similar to those reported in the United States (Bailey et al., 2014) but different to what have been reported in Japan (Minami et al., 2006). A study in India looking at rectal cancer using several registries have reported conflicting trends with most registries reporting decreasing or no change in the actual mean annual percentage change in men and the converse trend observed in female (Nooyi et al., 2014). These contrasted their findings based on the estimated changes. A study in Hong Kong, one of the developed nations in the East reported that increasing incidence of CRC in $\mathrm{HK}$ was mostly in the older and male population, but not in the younger age group (Yee et al., 2010). Similar factors as discussed earlier may account for these differences.

Our findings have several implications for both disease and healthcare burden and in the efforts to tackle the increasing problem of CRC. Even though the increase in the ASIRs in our population was small for the younger age groups, reflecting small increases in the absolute number, the trends are worrying. As our population continues to grow or when projected to a larger population, the actual number will be substantial. Similar trends are seen in colon cancers. If these trends are not addressed, there will be an increase in the absolute number of CRC and the number of young cancers will also increase. We have previously demonstrated that young CRC accounted for approximately one in eight of all CRC in our setting (Koh et al., 2015). It is important to address this issue given that this group of the population is economically active. Furthermore studies have shown that the stages of diseases are usually more advanced compared to the older group. There are studies that have shown that the treatment outcomes are favorable despite the stages of disease (Yeo et al., 2013). We are currently in the process of planning a national CRC screening program based on fecal occult blood testing and selective screening colonoscopy as the initial test. Based on our findings, the age of screening may need to be lower than the recommended age of 50 years above (Levin et al., 2008; Sung et al., 2015). However, this will increase the potential workload, straining the already stretched healthcare resource. Therefore, further studies are required to study the trends and also the best strategy to deal with the increasing trends including those in young CRC.

There are several limitations with our study. First the overall number of CRC cases is small and small fluctuations in the number of cases may cause wide fluctuations in the calculated ASR and ASIRs. However, the data captured by our registry is representative for the whole country and therefore, reflected the trends observed in our country. Secondly, the registry only captured histologically proven CRC cases diagnosed in the country and, therefore cases diagnosed outside of the country or without tissue diagnosis (i.e. radiologically) were not included. However, the number of such cases was likely to be very small and the trend of patients going outside of the country for investigations has been decreasing as the healthcare facilities improved. Finally, our study is only a single country study and the trends observed may not be generalized to another population, especially those with different demographic and racial backgrounds. However, despite these limitations, our findings are consistent with what have been reported in other different populations and also showed similarities to the trend reported in the neighboring South-East Asian nations.

In conclusion, our study showed that there was an increase in the incidence of CRC including in the younger age groups. The increase was seen earlier in rectal cancer compared to colon cancer. These mirror the trend reported from the West. Therefore, any health policies including CRC screening program dealing the chronic non-communicable diseases which include CRC need 
to take into account the disease pattern in the country. Future study will be required to assess if the increasing trends will continue and whether younger people will be affected with CRC.

\section{References}

Akbar A, Bhatti AB, Khattak S, et al (2014). Outcome of rectal cancer in patients aged 30 years or less in the Pakistani population. Asian Pac J Cancer Prev, 15, 6339-42.

Austin H, Jane Henley S, King J, Richardson LC, Eheman C (2014). Changes in colorectal cancer incidence rates in young and older adults in the United States: what does it tell us about screening. Cancer Causes Control, 25, 191-201.

Bailey CE, Hu CY, You YN (2015). Increasing disparities in the age-related incidences of colon and rectal cancers in the United States, 1975-2010. JAMA Surg, 150, 17-22.

Cai B, Wang MY, Liao K, et al (2014). Distribution characteristics of 3,369 Chinese colorectal cancer patients for gender, age, location and tumor size during colonoscopy. Asian Pac J Cancer Prev, 15, 8951-5.

Chong VH, Abdullah MS, Telisinghe PU, Jalihal A (2009). Colorectal cancer: incidence and trend in Brunei Darussalam. Singapore Med J, 50, 1085-9.

Chong VH, Bakar S, Sia R, et al (2013). Colorectal cancer screening among government servants in Brunei Darussalam. Asian Pac J Cancer Prev, 14, 7657-61.

Elsamany SA, Alzahrani AS, Mohamed MM (2014). Clinicopathological patterns and survival outcome of colorectal cancer in young patients: western Saudi Arabia experience. Asian Pac J Cancer Prev, 15, 5239-43.

International Agency for Research on Cancer (IARC). GLOBOCAN 2012. www.globocan.iarc.fr/ (accessed 15 $5^{\text {th }}$ April 2014).

Koh KS, Telisinghe PU, Bickle I, et al (2015). Characteristics of young colorectal cancer in Brunei Darussalam: Epidemiologic study over 30 years. Asian Pac J Cancer Prev [Epub ahead of print].

Levin B, Lieberman DA, McFarland B, et al (2008). Screening and surveillance for the early detection of colorectal cancer and adenomatous polyps, 2008: a joint guideline from the American cancer society, the US multi-society task force on colorectal cancer, and the American college of radiology. Gastroenterology, 134, 1570-95.

Lim KG (2014). A review of colorectal cancer research in Malaysia. Med J Malaysia, 69, 23-32.

Magaji BA, Moy FM, Roslani AC, Law CW (2014). Descriptive epidemiology of colorectal cancer in University Malaya Medical Centre, 2001 to 2010. Asian Pac J Cancer Prev, 15, 6059-64.

Minami Y, Nishino Y, Tsubono Y, Tsuji I, Hisamichi S (2006). Increase of colon and rectal cancer incidence rates in Japan: trends in incidence rates in Miyagi Prefecture, 1959-1997. J Epidemiol, 16, 240-8.

Nooyi SC, Murthy NS, Shivananjaiah S, Sreekantaiah P, Mathew A (2011). Trends in rectal cancer incidence-Indian scenario. Asian Pac J Cancer Prev, 12, 2001-6.

O'Connell JB, Maggard MA, Liu JH, et al (2003). Rates of colon and rectal cancers are increasing in young adults. $\mathrm{Am}$ Surg, 69, 866-72.

Sung JJ, Ng SC, Chan FK, et al (2015). An updated Asia Pacific Consensus Recommendations on colorectal cancer screening. Gut, 64, 121-32.

Yee YK, Gu Q, Hung I, Tan VP, et al (2010). Trend of colorectal cancer in Hong Kong: 1983-2006. J Gastroenterol Hepatol, 25, 923-7.

Yeo SA, Chew MH, Koh PK, Tang CL (2013). Young colorectal carcinoma patients do not have a poorer prognosis: a comparative review of 2,426 cases. Tech Coloproctol, 17, 653-61.

Zheng ZX, Zheng RS, Zhang SW, Chen WQ (2014). Colorectal cancer incidence and mortality in China, 2010. Asian Pac J Cancer Prev, 15, 8455-60. 